\title{
ERRATUM
}

\section{Erratum to: Should we take into account ESBLs in empirical antibiotic treatment?}

Matteo Bassetti ${ }^{1,3^{*}}$ and Jesús Rodríguez-Baño²

(C) 2016 Springer-Verlag Berlin Heidelberg and ESICM

\section{Erratum to: Intensive Care Med (2016) \\ 42:2059-2062 \\ DOI 10.1007/s00134-016-4599-6}

The given names and names of both authors were inadvertently transposed. Their correct names are (family names bold):

Matteo Bassetti and Jesús Rodríguez-Baño.

\section{Author details}

${ }^{1}$ Infectious Diseases Division, Santa Maria Misericordia University Hospital, Udine, Italy. ${ }^{2}$ Unidad Clínica Intercentros de Enfermedades Infecciosas, Microbiología Y Medicina Preventiva, Hospitales Universitarios Virgen Macarena Y Virgen Del Rocío-IbiS and Departamento de Medicina, Universidad de Sevilla, Seville, Spain. ${ }^{3}$ Clinica Malattie Infettive, Azienda Ospedaliero-Universitaria "Santa Maria Della Misericordia", Piazzale S. Maria Della Misericordian. 15, 33100 Udine, Italy.

Published online: 9 January 2017

\footnotetext{
*Correspondence: mattba@tin.it

${ }^{3}$ Clinica Malattie Infettive, Azienda Ospedaliero-Universitaria "Santa Maria Della Misericordia", Piazzale S. Maria Della Misericordian. 15, 33100 Udine, Italy

Full author information is available at the end of the article
}

The online version of the original article can be found under doi:10.1007/s00134-016-4599-6.

\section{Springer}

